\title{
ON JACOBI AND CONTINUOUS HAHN POLYNOMIALS
}

\author{
H. T. KOELINK
}

(Communicated by Hal L. Smith)

\begin{abstract}
Jacobi polynomials are mapped onto the continuous Hahn polynomials by the Fourier transform, and the orthogonality relations for the continuous Hahn polynomials then follow from the orthogonality relations for the Jacobi polynomials and the Parseval formula. In a special case this relation dates back to work by Bateman in 1933 and we follow a part of the historical development for these polynomials. Some applications of this relation are given.
\end{abstract}

\section{INTRODUCTION AND HISTORY}

In Askey's scheme of hypergeometric orthogonal polynomials we find the Jacobi polynomials and the continuous Hahn polynomials; see Askey and Wilson [5, Appendix] with the correction in [2], Koekoek and Swarttouw [20] or Koornwinder [23, $\S 5]$ for information on Askey's scheme. In the hierarchy of Askey's scheme of hypergeometric orthogonal polynomials the continuous Hahn polynomials are above the Jacobi polynomials since they have one extra degree of freedom. In this paper we consider a way of going up in the Askey scheme from the Jacobi polynomials to the continuous Hahn polynomials by use of the Fourier transform. This method is a simple extension of some special cases introduced by Bateman in the 1930's.

In his 1933 paper [9] Bateman introduced the polynomial $F_{n}$ satisfying

$$
F_{n}\left(\frac{d}{d x}\right) \cosh ^{-1} x=\cosh ^{-1} x P_{n}(\tanh x),
$$

where $P_{n}(x)=P_{n}^{(0,0)}(x)$ is the Legendre polynomial; cf. (2.3) for its definition. This is possible since $\frac{d}{d x} \cosh ^{-1}=-\cosh ^{-1} \tanh x$ and $\frac{d}{d x} \tanh x=1-\tanh ^{2} x$. Bateman derived in [9] various properties for the polynomials $F_{n}$, such as generating functions, explicit expressions as hypergeometric series, the three-term recurrence relation, difference equations, integral representations, and more; see also [10]. One year later Bateman proved the orthogonality relations (cf. [11], [10])

$$
\int_{-\infty}^{\infty} \frac{F_{n}(i x) F_{m}(i x)}{\cosh ^{2}(\pi x / 2)} d x=\delta_{n, m} \frac{4(-1)^{n}}{\pi(2 n+1)}
$$

Received by the editors September 28, 1994. 1991 Mathematics Subject Classification. Primary 33C45, 42A38.

Key words and phrases. Jacobi polynomials, continuous Hahn polynomials, Fourier transform. Supported by a Fellowship of the Research Council of the Katholieke Universiteit Leuven. 
by a method using the Fourier transform, which we reproduce for a more general class of polynomials. The factor $(-1)^{n}$ on the right-hand side of (1.2) does not matter, since it can be removed by rescaling $F_{n}$ by a factor $i^{n}$. This factor is necessary in order to make the polynomial $F_{n}$ real-valued for imaginary argument. The orthogonality relation (1.2) is also derived by Hardy [18, §8] as an example of a general approach to some orthogonal polynomials using the Mellin transform, which is equivalent to Bateman's proof of (1.2); see also Remark 3.2(ii).

The Bateman polynomial $F_{n}$ was generalised by Pasternack [26] in 1939. $\mathrm{He}$ defined the polynomial $F_{n}^{m}$ by

$$
F_{n}^{m}\left(\frac{d}{d x}\right) \cosh ^{-m-1} x=\cosh ^{-m-1} x P_{n}(\tanh x),
$$

for $m \in \mathbb{C} \backslash\{-1\}$, which reduces to Bateman's polynomial $F_{n}$ in case $m=0$. Here we use $\frac{d}{d x} \cosh ^{-1-m} x=-(m+1) \cosh ^{-1-m} x \tanh x$. The case $m=1$ already occurs in Bateman's paper [10, §4]; see also [12].

For the polynomials $F_{n}^{m}$ Pasternack derived explicit expressions in terms of hypergeometric series, generating functions, the three-term recurrence relation, difference equations, and integral representations much along the same lines as in [9], but he does not prove orthogonality relations for $F_{n}^{m}$. In particular, Pasternack proved (cf. [26, (10.2), (10.5)])

$$
\int_{-\infty}^{\infty}\left|\Gamma\left(\frac{1}{2}(m+1+z)\right)\right|{ }^{4} F_{n}^{m}(i z) F_{p}^{m}(-i z) d z=C \int_{-1}^{1}\left(1-x^{2}\right)^{m} P_{n}(x) P_{p}(x) d x
$$

for some explicit constant $C$. So if Pasternack would have replaced the Legendre polynomial $P_{n}$ in (1.3) by the Gegenbauer polynomial $P_{n}^{(m, m)}$, i.e. the polynomials orthogonal on $[-1,1]$ with respect to the weight function $\left(1-x^{2}\right)^{m}$ (cf. $(2.2)$ ), he would have obtained a one-parameter subclass of the two-parameter continuous symmetric Hahn polynomials introduced by Askey and Wilson [4] in 1982.

Bateman obtained in $[12,(3.3)]$ the orthogonality relations

$$
\int_{-\infty}^{\infty} \frac{F_{n}^{m}(i x) F_{p}^{-m}(i x)}{\cosh (\pi x)+\cos (m \pi)} d x=\delta_{n, p} \frac{2(-1)^{n}}{\pi(2 n+1)} \frac{m \pi}{\sin \pi m}, \quad-1<m<1,
$$

for Pasternack's polynomials, which reduce to (1.2) for $m=0$. The right-hand side of $[12,(3.3)]$ is not correct. Although explicit expressions for $F_{n}^{m}$, and hence for the leading coefficients of these polynomials, were known to Bateman at that time he did not rewrite the orthogonality relation (1.4) as the orthogonality relation (1.5) for the Pasternack polynomials.

In 1956 Touchard $[33, \S \S 13,14]$ considered orthogonal polynomials associated with the Bernoulli numbers. He derived a three-term recurrence relation and orthogonality relations and determined the value at $-\frac{1}{2}$. In the following paper in the Canadian Journal of Mathematics Wyman and Moser [36] gave an explicit expression for these polynomials in terms of a hypergeometric ${ }_{4} F_{3}$-series. A year later Brafman [13] gave another expression for these polynomials and derived generating functions for these polynomials. But it is Carlitz [14] who noted that the polynomials introduced by Touchard are the same as Bateman's polynomials $F_{n}$ defined by (1.1). In 1959 Carlitz [15] replaced the Bernoulli numbers by certain numbers 
involving the Bernoulli polynomials at a point $\lambda$, where the case $\lambda=0$ corresponds to the Bernoulli numbers; see also Chihara [16, Ch. VI, §8]. Carlitz showed that the corresponding orthogonal polynomials are Pasternack's polynomials (1.3), for which he gave the orthogonality relations (see also [4])

$$
\begin{aligned}
\int_{-\infty}^{\infty} & \frac{F_{n}^{m}(i x) F_{p}^{m}(i x)}{\cos (\pi m)+\cosh (\pi x)} d x \\
= & \delta_{n, p} \frac{(-1)^{n}}{2 n+1} \frac{2}{\pi} \frac{(1-m)_{n}}{(1+m)_{n}} \frac{m \pi}{\sin \pi m}, \quad-1<m<1,
\end{aligned}
$$

where we use the notation of (2.4). The same remark as for (1.2) applies here. The case $m=0$ of (1.5) is (1.2). From Proposition 3.1 we see that (1.5) also yields orthogonal polynomials, after a suitable renormalisation, for $m \in i \mathbb{R}$. From either (1.4) or from the fact that the weight function in (1.5) is even in $m$ we see that $F_{n}^{m}$ is a multiple of $F_{n}^{-m}$. By comparing leading coefficients (cf. $\S 2$ ), we obtain $(1+m)_{n} F_{n}^{m}(x)=(1-m)_{n} F_{n}^{-m}(x)$ (see $\S 2$ for the notation). The case $m=\frac{1}{2}$ of (1.5) was given by Hardy [18, $\S \S 4-7]$ in 1940.

Carlitz explicitly calculated the moments corresponding to the orthogonality measure (1.5) in terms of the Bernoulli polynomials; cf. [15, §6]. This result had already been obtained by Stieltjes $[29, \S 5]$ in 1890 by developing

$$
\psi(x+b)-\psi(x+1-b)
$$

$\psi(x)$ denoting the logarithmic derivative of the gamma function $\Gamma(x)$, in powers of $x^{-1}$ and in terms of a continued fraction. This means that Stieltjes gave the threeterm recurrence relation for the orthogonal polynomials for which the moments of the orthogonality measure are given in terms of the Bernoulli polynomials. Stieltjes included this example in his famous memoir "Recherches sur les fraction continues", in which he also gave the corresponding integral representation; cf. [30, §86].

In 1982 Askey and Wilson [4] introduced orthogonal polynomials, which generalise the orthogonal polynomials introduced by Bateman, Pasternack, Touchard, Hardy, and Carlitz. These polynomials are orthogonal on $\mathbb{R}$ with respect to the measure $|\Gamma(\alpha+i x) \Gamma(\gamma+i x)|^{2}(\alpha, \gamma>0$ or $\bar{\alpha}=\gamma$ and $\Re(\alpha)>0)$ and are nowadays known as the symmetric continuous Hahn polynomials. Atakishiyev and Suslov [6, $\S 3]$ showed that this is not the end of the story and introduced the continuous Hahn polynomials which have one extra parameter; see also Askey [2]. These polynomials are orthogonal with respect to the weight function $|\Gamma(\alpha+i x) \Gamma(\gamma+i x)|^{2}$ with $\Re(\alpha), \Re(\gamma)>0$ and $\Im(\alpha)=-\Im(\gamma)$.

The goal of this paper is to show that Bateman's approach can be used to prove the orthogonality relations for the continuous Hahn polynomials by only using the Jacobi polynomials and the Fourier transform in a similar way as Bateman [11] did in proving (1.2). The orthogonality relations for the continuous Hahn polynomials are not new, but this paper shows that Bateman, Pasternack, and Hardy could have found these orthogonality relations if they had pursued their approach somewhat further. Moreover, this point of view on the relationship between Jacobi polynomials and continuous Hahn polynomials gives an intrinsic explanation for the occurrence of the Jacobi polynomials in Atakishiyev and Suslov's proof $[6, \S 3]$ of the orthogonality relation for the continuous Hahn polynomials. 
We do not exactly know why Bateman and Hardy did not proceed to find the continuous Hahn polynomials as early as the 1930's and 1940's. The following explanation has been communicated to me by Richard Askey, whom I thank for letting me reproduce his view on this matter here. As to Hardy, we know that he kept the special functions, when needed, as simple as possible and that he only used special functions when necessary. So going beyond the Legendre polynomial was no option to Hardy. Bateman, as most of his contemporaries, thought of hypergeometric series as a function of the power series variable $z$, cf. the definition in $\S 2$. But the argument of the Bateman and Pasternack polynomial occurs in one of the parameters of a hypergeometric series (cf. Remark 2.2(i)), and not in the power series variable as is the case for e.g. the Jacobi polynomials, and thus they were not in the line of thought at that time. This also explains why Rice [28] looked for an appropriate generalisation of the Bateman and Pasternack polynomial by introducing a variable at the power series spot. However, Rice did not obtain orthogonal polynomials in this way.

There are more orthogonal systems involving orthogonal polynomials that are mapped onto each other by the Fourier transform, or by another integral transform such as the Mellin and Hankel transform. The best known examples of this are the Hermite functions, i.e. Hermite polynomials multiplied by $e^{-x^{2} / 2}$, which are eigenfunctions of the Fourier transform. For more examples we refer to Koornwinder [22], [23] and to the integral transforms of the Bateman project [17]. It is, however, important to note that in the derivation presented here we do not use orthogonal systems, but biorthogonal systems involving Jacobi polynomials. This gives the possibility to introduce the necessary extra degrees of freedom. Moreover, the result here is not a special case of Koornwinder's result in which the Jacobi polynomials are mapped onto the Wilson polynomials by use of the Jacobi function transform; cf. [22], [23].

A striking aspect of (1.1) and (1.3) is that the argument of the orthogonal polynomial is a differential operator. Badertscher and Koornwinder [7] (see also [23]) have given group-theoretic interpretations for several identities involving orthogonal polynomials of differential operator argument. In these cases these differential operators are acting on spherical functions on Riemannian symmetric spaces, which are usually more complicated special functions than just $\cosh ^{-1} x$. A related paper in this direction is [21].

The organisation of this paper is as follows. In $\S 2$ we derive the Fourier transform of certain Jacobi polynomials in terms of continuous Hahn polynomials and we discuss some applications. We also give the extension of (1.1) and (1.3), and we show how some properties of the continuous Hahn polynomials can be derived from properties of the Jacobi polynomials. In $\S 3$ we prove the orthogonality relation for the continuous Hahn polynomials from Parseval's identity for the Fourier transform.

\section{FOURIER TRANSFORM ON JACOBI POLYNOMIALS}

The gamma function was introduced by Euler in 1729 and is defined by

$$
\Gamma(z)=\int_{0}^{\infty} t^{z-1} e^{-t} d t, \quad \Re(z)>0 .
$$

The fundamental recurrence relation $\Gamma(z+1)=z \Gamma(z)$ follows by integration by 
parts. A closely related integral is the beta integral:

$$
B(\alpha, \beta)=\int_{0}^{1} t^{\alpha-1}(1-t)^{\beta-1} d t=\frac{\Gamma(\alpha) \Gamma(\beta)}{\Gamma(\alpha+\beta)}, \quad \Re(\alpha), \Re(\beta)>0 .
$$

The first proof of this result was given by Euler in 1772. More information on proofs for the beta integral and related integrals and sums as well as references to the literature can be found in the papers by Askey [3] and Rahman and Suslov [27].

The Jacobi polynomials $P_{n}^{(\alpha, \beta)}(x)$ of degree $n$ in $x$ are the orthogonal polynomials with respect to the beta measure shifted to the interval $[-1,1]$ :

$$
\begin{aligned}
\int_{-1}^{1}(1-x)^{\alpha}(1+x)^{\beta} P_{n}^{(\alpha, \beta)}(x) P_{m}^{(\alpha, \beta)}(x) d x & \\
& =\delta_{n, m} \frac{2^{\alpha+\beta+1}}{2 n+\alpha+\beta+1} \frac{\Gamma(n+\alpha+1) \Gamma(n+\beta+1)}{n ! \Gamma(n+\alpha+\beta+1)}
\end{aligned}
$$

for $\Re(\alpha)>-1, \Re(\beta)>-1$. The orthogonality relations are usually stated for $\alpha>-1, \beta>-1$, but they remain valid under this more general condition on the parameters $\alpha$ and $\beta$. The weight function is positive if and only if $\alpha$ and $\beta$ are real. An explicit expression for the Jacobi polynomial $P_{n}^{(\alpha, \beta)}(x)$ is given by a terminating hypergeometric series:

$$
P_{n}^{(\alpha, \beta)}(x)=\frac{(\alpha+1)_{n}}{n !}{ }_{2} F_{1}\left(\begin{array}{c}
-n, n+\alpha+\beta+1 \\
\alpha+1
\end{array} ; \frac{1-x}{2}\right)
$$

where the terminating hypergeometric series is defined by

$$
\begin{gathered}
{ }_{p+1} F_{p}\left(\begin{array}{c}
-n, a_{1}, \ldots, a_{p} \\
b_{1}, \ldots, b_{p}
\end{array} ;\right)=\sum_{k=0}^{n} \frac{(-n)_{k}\left(a_{1}\right)_{k} \ldots\left(a_{p}\right)_{k}}{\left(b_{1}\right)_{k} \ldots\left(b_{p}\right)_{k}} \frac{z^{k}}{k !}, \quad n \in \mathbb{Z}_{+}, \\
(a)_{k}=a(a+1)(a+2) \ldots(a+k-1)=\frac{\Gamma(a+k)}{\Gamma(a)}, \quad k \in \mathbb{Z}_{+} .
\end{gathered}
$$

More information on Jacobi polynomials can be found in Szegö's book [31, Ch. 4].

Let us calculate a Fourier transform involving Jacobi polynomials. Rewrite the Fourier transform

$$
\begin{aligned}
& \int_{-\infty}^{\infty} e^{-i x z}(1-\tanh x)^{\alpha}(1+\tanh x)^{\beta} P_{n}^{(\gamma, \delta)}(\tanh x) d x \\
& =\int_{-1}^{1}(1-t)^{\alpha-1+\frac{1}{2} i z}(1+t)^{\beta-1-\frac{1}{2} i z} P_{n}^{(\gamma, \delta)}(t) d t \\
& =2^{\alpha+\beta-1} \int_{0}^{1} u^{\alpha-1+\frac{1}{2} i z}(1-u)^{\beta-1-\frac{1}{2} i z} P_{n}^{(\gamma, \delta)}(1-2 u) d u
\end{aligned}
$$

using the substitutions $t=\tanh x, t=1-2 u$. Use that

$$
\frac{d t}{d x}=(\cosh x)^{-2}=(1-\tanh x)(1+\tanh x), \quad \frac{1-\tanh x}{1+\tanh x}=e^{-2 x} .
$$


Note that $x \mapsto(1-\tanh x)^{\alpha}(1+\tanh x)^{\beta} P_{n}^{(\gamma, \delta)}(\tanh x) \in L^{2}(\mathbb{R}) \cap L^{1}(\mathbb{R})$ for $\Re(\alpha), \Re(\beta)>0$.

In (2.5) we use the explicit series representation (2.3) for the Jacobi polynomial and the beta integral (2.1) to see that $(2.5)$ equals (2.6)

$$
\begin{aligned}
& 2^{\alpha+\beta-1} \frac{(\gamma+1)_{n}}{n !} \sum_{k=0}^{n} \frac{(-n)_{k}(n+\gamma+\delta+1)_{k}}{k !(\gamma+1)_{k}} B\left(\alpha+k+\frac{1}{2} i z, \beta-\frac{1}{2} i z\right) \\
& =2^{\alpha+\beta-1} \frac{(\gamma+1)_{n}}{n !} B\left(\alpha+\frac{1}{2} i z, \beta-\frac{1}{2} i z\right) \sum_{k=0}^{n} \frac{(-n)_{k}(n+\gamma+\delta+1)_{k}\left(\alpha+\frac{1}{2} i z\right)_{k}}{k !(\gamma+1)_{k}(\alpha+\beta)_{k}} \\
& =2^{\alpha+\beta-1} \frac{(\gamma+1)_{n}}{n !} B\left(\alpha+\frac{1}{2} i z, \beta-\frac{1}{2} i z\right)_{3} F_{2}\left(\begin{array}{c}
-n, n+\gamma+\delta+1, \alpha+\frac{1}{2} i z \\
\gamma+1
\end{array}\right) .
\end{aligned}
$$

The identity obtained in this way can also be found in Erdélyi et al. [17, 16.4(3)].

The ${ }_{3} F_{2}$-series in $(2.6)$ is a continuous Hahn polynomial defined by (cf. [2])

$$
\begin{aligned}
& p_{n}(x ; a, b, c, d) \\
& \quad=i^{n} \frac{(a+c)_{n}(a+d)_{n}}{n !}{ }_{3} F_{2}\left(\begin{array}{c}
-n, n+a+b+c+d-1, a+i x \\
a+c, a+d
\end{array} ; 1\right) .
\end{aligned}
$$

So we have proved the following lemma.

Lemma 2.1. For $z \in \mathbb{R}, \Re(\alpha), \Re(\beta)>0$, and $-\gamma \notin \mathbb{N}$ we have

$$
\begin{aligned}
& \int_{-\infty}^{\infty} e^{-i x z}(1-\tanh x)^{\alpha}(1+\tanh x)^{\beta} P_{n}^{(\gamma, \delta)}(\tanh x) d x \\
& \quad=2^{\alpha+\beta-1} \frac{\Gamma\left(\alpha+\frac{1}{2} i z\right) \Gamma\left(\beta-\frac{1}{2} i z\right)}{\Gamma(\alpha+\beta+n)} i^{-n} p_{n}(z / 2 ; \alpha, \delta-\beta+1, \gamma-\alpha+1, \beta),
\end{aligned}
$$

where $P_{n}^{(\gamma, \delta)}(x)$ is a Jacobi polynomial defined by $(2.3)$ and $p_{n}(x ; a, b, c, d)$ is a continuous Hahn polynomial defined by (2.7).

Remark 2.2. (i) An equivalent way of proving the lemma is by establishing

$$
\begin{aligned}
p_{n}(- & \left.\frac{i}{2} \frac{d}{d x} ; \alpha, \delta-\beta+1, \gamma-\alpha+1, \beta\right)\left\{(1-\tanh x)^{\alpha}(1+\tanh x)^{\beta}\right\} \\
= & i^{n}(\alpha+\beta)_{n}\left\{(1-\tanh x)^{\alpha}(1+\tanh x)^{\beta}\right\} P_{n}^{(\gamma, \delta)}(\tanh x)
\end{aligned}
$$

and applying the Fourier transform $\mathcal{F}$ to it and using $\mathcal{F}\left(f^{\prime}\right)(z)=i z \mathcal{F}(f)(z)$. Equation (2.8) can be proved from

$$
\begin{aligned}
& \frac{d}{d x}\left[(1-\tanh x)^{\alpha}(1+\tanh x)^{\beta}\right] \\
& \quad=(\alpha+\beta+(\alpha-\beta) \tanh x)\left[(1-\tanh x)^{\alpha}(1+\tanh x)^{\beta}\right], \\
& \left(\alpha+\frac{1}{2} \frac{d}{d x}\right)_{r}\left[(1-\tanh x)^{\alpha}[1+\tanh x)^{\beta}\right] \\
& \quad=2^{-r}(1-\tanh x)^{r}(\alpha+\beta)_{r}\left[(1-\tanh x)^{\alpha}(1+\tanh x)^{\beta}\right],
\end{aligned}
$$


which in turn can be proved by induction with respect to $r \in \mathbb{Z}_{+}$. Special cases of (2.8) are (1.1) for $\alpha=\beta=\frac{1}{2}, \gamma=\delta=0$ and (1.3) for $\alpha=\beta=\frac{1}{2}(m+1), \gamma=\delta=0$ for the Bateman polynomial and its generalisation by Pasternack. So we have

$$
\begin{aligned}
F_{n}^{m}(x) & =\frac{1}{i^{n}(m+1)_{n}} p_{n}\left(-\frac{i}{2} x ; \frac{1}{2}(1+m), \frac{1}{2}(1-m), \frac{1}{2}(1-m), \frac{1}{2}(1+m)\right) \\
& ={ }_{3} F_{2}\left(\begin{array}{c}
-n, n+1, \frac{1}{2}(1+m+x) \\
1, m+1
\end{array} ; 1\right)
\end{aligned}
$$

for the Bateman $(m=0)$ and Pasternack polynomials defined in (1.1) and (1.3). The case $\alpha=\beta=\frac{1}{4}+\frac{i}{2} \lambda, \gamma=\delta=-\frac{1}{2}$ of (2.8) was observed by Koornwinder [23, $\S 2]$.

(ii) Applying the inverse Fourier transform and taking $n=0$ gives the Fourier transform of $z \mapsto \Gamma(\alpha+i z) \Gamma(\beta-i z)$, which is closely related to the orthogonality measure for the Meixner-Pollaczek polynomials; cf. Askey [3].

Lemma 2.1 can be used to obtain identities for the continuous Hahn polynomials from identities satisfied by the Jacobi polynomials. As a first example we start with the following generating functions for the Jacobi polynomials (cf. [20, (1.8.7), $(1.8 .6)])$ :

$$
\begin{gathered}
(1-t)^{-\gamma-\delta-1}{ }_{2} F_{1}\left(\begin{array}{c}
\frac{1}{2}(\gamma+\delta+1), \frac{1}{2}(\gamma+\delta+2) \\
\gamma+1
\end{array} ; \frac{2(x-1) t}{(1-t)^{2}}\right) \\
=\sum_{n=0}^{\infty} \frac{(\gamma+\delta+1)_{n}}{(\gamma+1)_{n}} P_{n}^{(\gamma, \delta)}(x) t^{n}
\end{gathered}
$$

and

$$
{ }_{0} F_{1}\left(\begin{array}{c}
- \\
\gamma+1
\end{array} ; \frac{(x-1) t}{2}\right){ }_{0} F_{1}\left(\begin{array}{c}
- \\
\delta+1
\end{array} ; \frac{(x+1) t}{2}\right)=\sum_{n=0}^{\infty} \frac{P_{n}^{(\gamma, \delta)}(x) t^{n}}{(\gamma+1)_{n}(\delta+1)_{n}}
$$

A straightforward manipulation using Lemma 2.1 proves the following generating functions for the continuous Hahn polynomials. The first of these generating functions is also contained in $[20,(1.4 .8)]$. We get

$$
\begin{aligned}
& (1-t)^{-\alpha-\beta-\gamma-\delta-1} \\
& \quad \times{ }_{3} F_{2}\left(\begin{array}{c}
\frac{1}{2}(\alpha+\beta+\gamma+\delta-1), \frac{1}{2}(\alpha+\beta+\gamma+\delta), \alpha+i z \\
\gamma+\alpha, \quad \alpha+\beta
\end{array} ; \frac{-4 t}{(1-t)^{2}}\right) \\
& \quad=\sum_{n=0}^{\infty} \frac{(\alpha+\beta+\gamma+\delta-1)_{n}}{(\alpha+\beta)_{n}(\alpha+\gamma)_{n}}(t / i)^{n} p_{n}(z ; \alpha, \delta, \gamma, \beta),
\end{aligned}
$$

which is the generating function used by Bateman $[9, \S 3]$ and Pasternack $[26,(2.2)]$, and

$$
\sum_{n=0}^{\infty} \frac{(t / i)^{n} p_{n}(z ; \alpha, \delta, \gamma, \beta)}{(\gamma+\alpha)_{n}(\delta+\beta)_{n}(\alpha+\beta)_{n}}=\sum_{p=0}^{\infty} \sum_{k=0}^{\infty} \frac{(-t)^{p} t^{k}(\alpha+i z)_{p}(\beta-i z)_{k}}{p !(\gamma+\alpha)_{p} k !(\delta+\beta)_{k}(\alpha+\beta)_{p+k}}
$$


The last series can be rewritten as a hypergeometric series in two variables with arguments $x=-t, y=t$, cf. Appell and Kampé de Fériet [1, Ch. IX, p. 150, (29)].

As a second example we derive two relations between three continuous Hahn polynomials from identities for Jacobi polynomials. First use $\mathcal{F}\left(f^{\prime}\right)(z)=i z \mathcal{F}(f)(z)$, (2.9) and $\frac{d}{d x} P^{(\gamma, \delta)}(x)=\frac{1}{2}(n+\gamma+\delta+1) P_{n-1}^{(\gamma+1, \delta+1)}(x)$ (cf. [31, (4.21.7)]), and straightforward calculations to get after some rewriting

$$
\begin{aligned}
& (\alpha+\beta+n) i z p_{n}(z ; \alpha, \delta, \gamma, \beta)=(\alpha+\beta)(\alpha+i z) p_{n}(z ; \alpha+1, \delta, \gamma-1, \beta) \\
& +i(n+\alpha+\beta+\gamma+\delta-1)(\alpha+i z)(\beta-i z) p_{n-1}(z ; \alpha+1, \delta, \gamma, \beta+1)
\end{aligned}
$$

Another classical identity for the Jacobi polynomials (cf. [31, (4.5.4)])

$$
(n+\gamma+1) P_{n}^{(\gamma, \delta)}(x)-(n+1) P_{n+1}^{(\gamma, \delta)}(x)=\frac{1}{2}(2 n+\gamma+\delta+2)(1-x) P_{n}^{(\gamma+1, \delta)}(x)
$$

leads to

$$
\begin{aligned}
& (2 n+\alpha+\beta+\gamma+\delta)(\alpha+i z) p_{n}(z ; \alpha+1, \delta, \gamma, \beta) \\
& \quad=(\alpha+\beta+n)(n+\gamma+\alpha) p_{n}(z ; \alpha, \delta, \gamma, \beta)+i(n+1) p_{n+1}(z ; \alpha, \delta, \gamma, \beta)
\end{aligned}
$$

It is also possible to use two or more identities for the Jacobi polynomials in order to obtain identities for continuous Hahn polynomials. As an example we indicate how the three-term recurrence relation for the continuous Hahn polynomials can be derived; cf. Pasternack $[26, \S 5]$. Let $p_{n}$ (cf. (2.8)) be defined by (2.10)

$p_{n}\left(\frac{d}{d x}\right)\left\{(1-\tanh x)^{\alpha}(1+\tanh x)^{\beta}\right\}=\left\{(1-\tanh x)^{\alpha}(1+\tanh x)^{\beta}\right\} P_{n}^{(\gamma, \delta)}(\tanh x)$.

Differentiate this identity once more to get

$$
\begin{aligned}
& \frac{d}{d x} p_{n}\left(\frac{d}{d x}\right)\left\{(1-\tanh x)^{\alpha}(1+\tanh x)^{\beta}\right\}=\left\{(1-\tanh x)^{\alpha}(1+\tanh x)^{\beta}\right\} \\
& \quad \times\left[(\alpha+\beta+(\alpha-\beta) \tanh x) P_{n}^{(\gamma, \delta)}(\tanh x)+\left(1-\tanh ^{2} x\right) \frac{d P_{n}^{(\gamma, \delta)}}{d x}(\tanh x)\right] .
\end{aligned}
$$

In the term in square brackets we use

$$
\left(1-x^{2}\right) \frac{d P_{n}^{(\gamma, \delta)}}{d x}(x)=A_{n} P_{n+1}^{(\gamma, \delta)}(x)+B_{n} P_{n}^{(\gamma, \delta)}(x)+C_{n} P_{n-1}^{(\gamma, \delta)}(x)
$$

(cf. Szegö $[31,(4.5 .5)]$ for the explicit values of the constants) and the three-term recurrence relation for the Jacobi polynomials (cf. $[31,(4.5 .1)]$ ) to get only Jacobi polynomials of degree $n+1, n$, and $n-1$. Recalling (2.10) we find the three-term recurrence relation for the continuous Hahn polynomials $p_{n}$. For the explicit values of the coefficients we refer to $[20,(1.4 .3)]$. 


\section{Orthogonality For the CONTINuOUs Hahn POlynomials}

The set of functions $x \mapsto(1-\tanh x)^{\alpha}(1+\tanh x)^{\beta} P_{n}^{(2 \alpha+1,2 \beta+1)}$ is an orthogonal basis of $L^{2}(\mathbb{R})$, and by Lemma 2.1 it is mapped by the Fourier transform onto the set of functions $z \mapsto \Gamma\left(\alpha+\frac{1}{2} i z\right) \Gamma\left(\beta-\frac{1}{2} i z\right) p_{n}\left(\frac{1}{2} z ; \alpha, \beta, \alpha, \beta\right)$. Since the Fourier transform is isometric, we obtain the orthogonality relations for the continuous symmetric Hahn polynomials (cf. [4], [22], [23]), but we can do better as follows.

From the Parseval identity $2 \pi \int_{\mathbb{R}} f(x) \bar{g}(x) d x=\int_{\mathbb{R}}(\mathcal{F} f)(z) \overline{(\mathcal{F} g)}(z) d z$ for the Fourier transform for $f, g \in L^{2}(\mathbb{R})$ we obtain

$$
\begin{aligned}
& 2 \pi \int_{-\infty}^{\infty}(1-\tanh x)^{\alpha+a}(1+\tanh x)^{\beta+b} P_{n}^{(\gamma, \delta)}(\tanh x) P_{m}^{(c, d)}(\tanh x) d x \\
& =i^{m-n} 2^{\alpha+a+\beta+b-2} \int_{-\infty}^{\infty} \frac{\Gamma\left(\alpha+\frac{1}{2} i z\right) \Gamma\left(\beta-\frac{1}{2} i z\right)}{\Gamma(\alpha+\beta+n)} \frac{\Gamma\left(a-\frac{1}{2} i z\right) \Gamma\left(b+\frac{1}{2} i z\right)}{\Gamma(a+b+m)} \\
& \quad \times p_{n}(z / 2 ; \alpha, \delta-\beta+1, \gamma-\alpha+1, \beta) \\
& \quad \times \frac{p_{m}(z / 2 ; \bar{a}, \bar{d}-\bar{b}+1, \bar{c}-\bar{a}+1, \bar{b})}{\Gamma} d z
\end{aligned}
$$

for $\Re(\alpha), \Re(\beta), \Re(a), \Re(b)>0$. Next we restrict the parameters in the left-hand side of (3.1) such that we get the orthogonality relations (2.2) for the Jacobi polynomials. So we take $\Re(\alpha+a)>0, \Re(\beta+b)>0$, which is already satisfied, and $\gamma=c=$ $\alpha+a-1, \delta=d=\beta+b-1$. For these choices we put again $t=\tanh x$ to see that the left-hand side (3.1) equals zero for $n \neq m$. The square norm follows from $(2.2)$ and so we get

$$
\begin{aligned}
& \int_{-\infty}^{\infty} \Gamma(\alpha+i z) \Gamma(\beta-i z) \Gamma(a-i z) \Gamma(b+i z) \\
& \quad \times p_{n}(z ; \alpha, b, a, \beta) \overline{p_{m}(z ; \bar{a}, \bar{\beta}, \bar{\alpha}, \bar{b})} d z \\
& \quad=2 \pi \delta_{n, m} \frac{\Gamma(\alpha+\beta+n) \Gamma(a+b+n) \Gamma(n+\alpha+a) \Gamma(n+\beta+b)}{n !(2 n+\alpha+\beta+a+b-1) \Gamma(n+\alpha+\beta+a+b-1)}
\end{aligned}
$$

subject to the conditions $\Re(\alpha), \Re(\beta), \Re(a), \Re(b)>0$, since $\gamma=c=\alpha+a-1$, $\delta=d=\beta+b-1$. If we replace $p_{m}$ in (3.2) by $l c\left(p_{m}\right) z^{m}$, then (3.2) remains valid for $0 \leq m \leq n$. Here $l c\left(p_{m}\right)$ denotes the leading coefficient of $\overline{p_{m}(z ; \bar{a}, \bar{\beta}, \bar{\alpha}, \bar{b})}$, and since this equals the leading coefficient of $p_{n}(z ; \alpha, b, a, \beta)$ (cf. (2.7)), we may replace $\overline{p_{m}(z ; \bar{a}, \bar{\beta}, \bar{\alpha}, \bar{b})}$ by $p_{m}(z ; \alpha, b, a, \beta)$. So we have proved the orthogonality relations for the continuous Hahn polynomials stated in the next proposition from the Parseval identity for the Fourier transform and from the explicit knowledge of the Fourier transform of the Jacobi polynomial described in Lemma 2.1.

Proposition 3.1. The continuous Hahn polynomials defined in (2.7) satisfy

$$
\begin{aligned}
\frac{1}{2 \pi} \int_{-\infty}^{\infty} & \Gamma(\alpha+i z) \Gamma(\beta-i z) \Gamma(a-i z) \Gamma(b+i z) \\
& \times p_{n}(z ; \alpha, b, a, \beta) p_{m}(z ; \alpha, b, a, \beta) d z \\
& =\delta_{n, m} \frac{\Gamma(\alpha+\beta+n) \Gamma(a+b+n) \Gamma(n+\alpha+a) \Gamma(n+\beta+b)}{n !(2 n+\alpha+\beta+a+b-1) \Gamma(n+\alpha+\beta+a+b-1)}
\end{aligned}
$$

for $\Re(\alpha), \Re(\beta), \Re(a), \Re(b)>0$. 
Remark 3.2. (i) The weight function is positive for $\bar{a}=\alpha, \bar{b}=\beta$, or for $\alpha=\bar{\beta}$, $a=\bar{b}$, which follows from the invariance of (3.3), interchanging $\alpha$ and $b$ or $\beta$ and $a$.

(ii) The case $n=m=0$ of (3.3) is Barnes' first lemma from 1908; see e.g. Bailey $[8,1.7]$ or Whittaker and Watson $[34, \S 14.52]$ for the original proof by Barnes. An equivalent proof of Barnes' first lemma as given here can be found in Titchmarsh's book $[32,(7.8 .3)]$, where the Mellin transform is used instead of the Fourier transform. Proposition 3.1 can be obtained in a similar way using the Mellin transform if we use the Jacobi polynomials of argument $(1-x) /(1+x)$. We obtain an orthogonal system on $[0, \infty)$. So we start with the Mellin transform

$$
\begin{aligned}
\int_{0}^{\infty} & \frac{x^{\alpha}}{(1+x)^{\alpha+\beta}} P_{n}^{(\gamma, \delta)}\left(\frac{1-x}{1+x}\right) x^{-i \lambda-1} d x \\
\quad & =\frac{\Gamma(\alpha-i \lambda) \Gamma(\beta-i \lambda)}{\Gamma(\alpha+\beta+n)} i^{-n} p_{n}(-\lambda ; \alpha, \delta-\beta+1, \gamma-\alpha+1, \beta)
\end{aligned}
$$

and the Parseval formula for the Mellin transform gives Proposition 3.1. See Hardy $[18, \S 8]$ for this approach to Bateman's polynomial $F_{n}$. In [24, Prop. 3.1] Koornwinder shows that the Laguerre polynomials are mapped onto the MeixnerPollaczek polynomials by the Mellin transform. The Mellin transform of the underlying measures is given in Titchmarsh [32, (7.8.1)]. This is a limiting case of (3.4). Replace in (3.4) $x$ by $x / \delta$ and $\beta$ by $\delta \xi$ with $\Re(\xi)>0,|\Im(\xi)|<\pi$ and let $\delta \rightarrow \infty$. Similarly we can obtain the analogue of Lemma 2.1 with the Laguerre and Meixner-Pollaczek polynomials by a suitable limit transition.

(iii) The result (3.3) in this form has been proved first by Askey [2] using Barnes' first lemma and the Chu-Vandermonde summation formula for a terminating ${ }_{2} F_{1}$ series of unit argument. Before that Atakishiyev and Suslov [6] (see also [25, $\S 3.10 .3 .2]$ ) proved (3.3) in the case of a positive weight function. The method employed by Atakishiyev and Suslov uses Barnes' first lemma, which is rewritten in terms of the beta integral so that the orthogonality relations of the Jacobi polynomials can be used.

(iv) Another proof of Proposition 3.1 using symmetry in the parameters $a, b, \alpha$, $\beta$ was given by Kalnins and Miller $[19, \S 3]$. They also gave a proof of Barnes' first lemma in this way.

(v) The continuous Hahn polynomials are not on the top shelf of the Askey scheme of hypergeometric polynomials. The most general hypergeometric orthogonal polynomials with a continuous weight function are the Wilson polynomials (cf. Wilson [35]), which have four degrees of freedom. The continuous Hahn polynomials can be obtained from the Wilson polynomials by a suitable limit process; $\mathrm{cf}$. e.g. $[20, \S 2.2]$.

The orthogonality relations stated in $\S 1$ are special cases of (3.3). In particular, the orthogonality (1.5) for the Pasternack polynomials follows by taking $\alpha=\beta=$ $\frac{1}{2}(1+m), a=b=\frac{1}{2}(1-m)$. This shows also that we have a positive weight function for $-1<m<1$ or $m \in i \mathbb{R}$, which are equivalent for $m$ and $-m$. To see this we have to use the reflection formula $\Gamma(z) \Gamma(1-z)=\pi \sin ^{-1}(\pi z)$ and some straightforward manipulations on goniometric and hyperbolic functions. It should also be noted that taking the same values for the parameters in (3.2) gives Bateman's (bi)orthogonality relations (1.4). 


\section{ACKNOWLEDGMENT}

The work for this paper was initiated by the referee report for [21], in which the papers by Bateman and Pasternack were mentioned. I thank the referee for drawing my attention to these papers.

\section{REFERENCES}

1. P. Appell and J. Kampé de Fériet, Fonctions hypergéométriques et hypersphériques. Polynomes d'Hermite, Gauthier-Villars, Paris, 1926.

2. R. Askey, Continuous Hahn polynomials, J. Math. Phys. A: Math. Gen. 18 (1985), L1017L1019. MR 87d:33021

3. _ Beta integrals and associated orthogonal polynomials, Number Theory (K. Alladi, ed.), Lecture Notes in Math., vol. 1395, Springer-Verlag, New York, 1989, pp. 84-121. MR 90k:33001

4. R. Askey and J. Wilson, A set of hypergeometric orthogonal polynomials, SIAM J. Math. Anal. 13 (1982), 651-655. MR 83h:33010

5. __ Some basic hypergeometric orthogonal polynomials that generalize Jacobi polynomials, Mem. Amer. Math. Soc.,vol. 54, no. 319 (1985), Amer. Math. Soc., Providence, RI. MR 87a:05023

6. N.M. Atakishiyev and S.K. Suslov, The Hahn and Meixner polynomials of imaginary argument and some of their applications, J. Math. Phys. A: Math. Gen. 18 (1985), 1583-1596. MR 87i:33021

7. E. Badertscher and T.H. Koornwinder, Continuous Hahn polynomials of differential operator argument and analysis on Riemannian symmetric spaces of constant curvature, Canad. J. Math. 44 (1992), 750-773. MR 94k:43011

8. W.N. Bailey, Generalized hypergeometric series, Cambridge Tracts 32, Cambridge University Press, London and New York, 1935; reprinted by Hafner Publishing Company, New York, 1972. MR 32:2625

9. H. Bateman, Some properties of a certain set of polynomials, Tôhoku Math. J. 37 (1933) $23-38$.

10.

11. Functions orthogonal in the Hermitean sense. A new application of basic numbers, Proc. Nat. Acad. Sci. U.S.A. 20 (1934), 63-66.

12. _ An orthogonal property of the hypergeometric polynomial, Proc. Nat. Acad. Sci U.S.A. 28 (1942), 374-377. MR 4:83b

13. F. Brafman, On Touchard polynomials, Canad. J. Math. 9 (1957), 191-193. MR 19:28a

14. L. Carlitz, Some polynomials of Touchard connected with the Bernoulli numbers, Canad. J. Math. 9 (1957), 188-190. MR 19:27e

15. _ Bernoulli and Euler numbers and orthogonal polynomials, Duke Math. J. 26 (1959), 1-15. MR 21:2761

16. T.S. Chihara, An introduction to orthogonal polynomials, Math. Appl., vol. 13, Gordon and Breach, New York, 1978. MR 58:1979

17. A. Erdélyi, W. Magnus, F. Oberhettinger and F.G. Tricomi, Tables of integral transforms, Vol. 2, McGraw-Hill, New York, 1954. MR 16:468e

18. G.H. Hardy, Notes on special systems of orthogonal functions (III): a system of orthogonal polynomials, Proc. Cambridge Philos. Soc. 36 (1940), 1-8; reprinted in Collected Papers, vol. IV (1969), Oxford University Press, London and New York, pp. 552-559. MR 1:141c

19. E.G. Kalnins and W. Miller, q-Series and orthogonal polynomials associated with Barnes' first lemma, SIAM J. Math. Anal. 19 (1988), 1216-1231. MR 89m:33018

20. R. Koekoek and R.F. Swarttouw, The Askey-scheme of orthogonal polynomials and its qanalogue, Report 94-05, Technical University Delft (1994).

21. H.T. Koelink, Identities for q-ultraspherical polynomials and Jacobi functions, Proc. Amer. Math. Soc. 123 (1995), 2479-2487.

22. T.H. Koornwinder, Special orthogonal polynomial systems mapped onto each other by the Fourier-Jacobi transfom, Polynômes Orthogonaux et Applications (C. Brezinski, A. Draux, A.P. Magnus, P. Maroni and A. Ronveaux, eds.), Lecture Notes in Math., vol. 1171, SpringerVerlag, 1985, pp. 174-183. MR 87g:33007 
23. Group theoretic interpretations of Askey's scheme of hypergeometric orthogonal polynomials, Orthogonal Polynomials and their Applications (M. Alfaro, J.S. Dehesa, F.J. Marcellan, J.L. Rubio de Francia and J. Vinuesa, eds.), Lecture Notes in Math., vol. 1329, SpringerVerlag, 1988, pp. 46-72. MR 90b:33024

24. , Meixner-Pollaczek polynomials and the Heisenberg algebra, J. Math. Phys. 30 (1989), 767-769. MR 90e:33037

25. A.F. Nikiforov, S.K. Suslov and V.B. Uvarov, Classical orthogonal polynomials of a discrete variable, Springer Ser. Comput. Phys., Springer-Verlag, New York and Berlin, 1991. MR 92m:33019

26. S. Pasternack, A generalisation of the polynomial $F_{n}(x)$, London, Edinburgh, Dublin Philosophical Magazine and J. Science, Ser. 728 (1939), 209-226. MR 1:116a

27. M. Rahman and S.K. Suslov, The Pearson integral and the beta integrals, SIAM J. Math. Anal. 25 (1994), 646-693. MR 95f:33001

28. S.O. Rice, Some properties of ${ }_{3} F_{2}(-n, n+1, \zeta ; 1, p ; v)$, Duke Math. J. 6 (1940), 108-119. MR 1:234b

29. T.J. Stieltjes, Sur quelques intégrales définies et leur développement en fractions continues, Quart. J. Math., London 24 (1890), 370-382; reprinted in Euvres Complètes-Collected Papers, vol. II (G. van Dijk, ed.), Springer-Verlag, New York, 1993, pp. 382-395. MR 95g:01033

30. Recherches sur les fractions continues, Annales de la Faculté des Sciences de Toulouse 8 (1894), J.1-122, 9 (1895), A.1-47; reprinted in Euvres Complètes-Collected Papers, vol. II (G. van Dijk, ed.), Springer-Verlag, New York, 1993, pp. 406-570. MR 95g:01033

31. G. Szegö, Orthogonal polynomials, Colloq. Publ., vol. 23, 4th ed., Amer. Math. Soc., Providence, RI, 1975. MR 51:8724

32. E.C. Titchmarsh, Introduction to the theory of Fourier integrals, 2nd ed., Oxford University Press, New York, 1948.

33. J. Touchard, Nombres exponentiels et nombres de Bernoulli, Canad. J. Math. 8 (1956), 305320. MR 18:16f

34. E.T. Whittaker and G.N. Watson, A course of modern analysis, 4th ed., Cambridge University Press, London and New York, 1927.

35. J.A. Wilson, Some hypergeometric orthogonal polynomials, SIAM J. Math. Anal. 11 (1980), 690-701. MR 82a:33014

36. M. Wyman and L. Moser, On some polynomials of Touchard, Canad. J. Math. 8 (1956), 321-322. MR 18:17a

Department of Mathematics, Katholieke Universiteit Leuven, Celestijnenlaan 200 B, B-3001 Leuven (Heverlee), Belgium

Current address: Department of Mathematics, Universiteit van Amsterdam, Plantage Muidergracht 24, 1018 TV Amsterdam, the Netherlands

E-mail address: koelink@fwi.uva.nl 